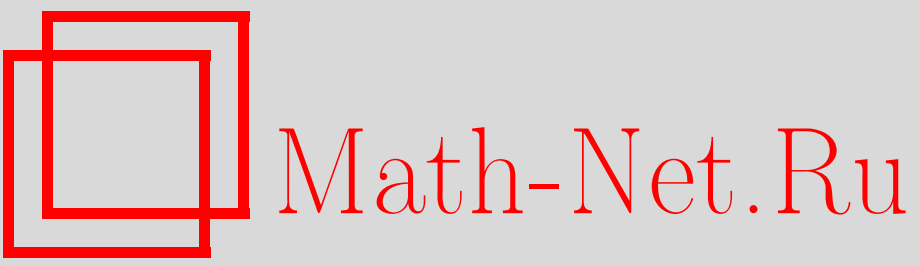

Д. Н. Попова, В. Е. Зотеев, Разработка и исследование линейно параметрической дискретной модели амплитудно-частотной характеристики механической системы с линейно-вязким трением, Вестн. Сам. гос. техн. ун-та. Сер. Физ.мат. науки, 2007, выпуск 2(), 179-182

DOI: https://doi.org/10.14498/vsgtu554

Использование Общероссийского математического портала Math-Net.Ru подразумевает, что вы прочитали и согласны с пользовательским соглашением

http://www.mathnet.ru/rus/agreement

Параметры загрузки:

IP : 54.209 .52 .79

26 апреля 2023 г., 12:21:59 


\title{
УДК 519.246
}

\author{
Д. Н. Попова, В. Е. Зотеев
}

\section{РАЗРАБОТКА И ИССЛЕДОВАНИЕ ЛИНЕЙНО ПАРАМЕТРИЧЕСКОЙ ДИСКРЕТНОЙ МОДЕЛИ АМПЛИТУДНО-ЧАСТОТНОЙ ХАРАКТЕРИСТИКИ МЕХАНИЧЕСКОЙ СИСТЕМЫ С ЛИНЕЙНО-ВЯЗКИМ ТРЕНИЕМ}

Рассматривается построение линейно параметрической дискретной модели в форме стохастического разностного уравнения, описывающей последовательность результатов измерений амплитудно-частотной характеристики диссипативной систелы с линейно-вязким трением. Предлагается численный итераиионный метод среднеквадратичного оценивания коэбфбииентов стохастического разностного уравнения. Проводится сравнительный анализ предлагаемого алгоритма вычисления диссипативной характеристики системы с известным методом кривой резонанся.

Рассматривается задача определения декремента колебаний по амплитудно-частотной характеристике (АЧХ) механических систем с линейно-вязким трением. В существующих методах определения декремента колебаний по АЧХ, например методе кривой резонанса [1], отсутствует возможность статистической обработки данных эксперимента и, как следствие, помехозащищённость результатов вычислений мала. В условиях промышленного эксперимента, когда случайная помеха в результатах измерения мгновенных значений АЧХ достаточно велика, погрешность оценок декремента колебаний может оказаться недопустимо высокой. Повысить точность определения декремента колебаний можно с помощью принципиально новых алгоритмов обработки результатов измерения мгновенных значений АЧХ, в основе которых лежат линейно параметрические дискретные модели (ЛПДМ) в форме стохастических разностных уравнений, описывающих результаты наблюдений [2].

Дифрференциальное уравнение, описывающее вынужденные гармонические колебания диссипативной системы с линейно-вязким трением, может быть представлено в виде $y^{\prime \prime}(t)+2 \delta y^{\prime}(t)+$ $+p^{2} y(t)=P_{0} \sin \omega t$, где $p$ и $\delta-$ резонансная частота и декремент колебаний, $\omega$ и $P_{0}-$ частота и приведённая к массе системы амплитуда внешней гармонической силы. Решением этого уравнения являются установившиеся колебания, зависимость амплитуды которых от частоты возбуждения (АЧХ системы) описывается фрункцией вида [3]

$$
\tilde{a}(\omega)=\frac{P_{0}}{\sqrt{\left(p^{2}-\omega^{2}\right)^{2}+4 \delta^{2} \omega^{2}}} .
$$

При равномерной с периодом $\Delta \omega$ дискретизации функции (1) получаем ее дискретной аналог $\tilde{a}_{k}=\frac{P_{0}}{\sqrt{\left(p^{2}-\Delta \omega^{2} k^{2}\right)^{2}+4 \delta^{2} \Delta \omega^{2} k^{2}}}$, который описывает значения АЧХ в точках $\omega_{k}=\Delta \omega k(k=0,1,2, \ldots)$. Отсюда получаем $\tilde{a}_{k}^{2}\left[\left(p^{2}-\Delta \omega^{2} k^{2}\right)^{2}+4 \delta^{2} \Delta \omega^{2} k^{2}\right]=P_{0}^{2}$. Применение $z$-преобразования к обеим частям данного выражения даёт $Z\left\{\tilde{a}_{k}^{2}\left[\left(p^{2}-\Delta \omega^{2} k^{2}\right)^{2}+4 \delta^{2} \Delta \omega^{2} k^{2}\right]\right\}=\frac{P_{0}^{2}}{1-z^{-1}}$. Отсюда имеем

$$
Z\left\{\tilde{a}_{k}^{2}\left[\left(p^{2}-\Delta \omega^{2} k^{2}\right)^{2}+4 \delta^{2} \Delta \omega^{2} k^{2}\right]\right\}-z^{-1} Z\left\{\tilde{a}_{k}^{2}\left[\left(p^{2}-\Delta \omega^{2} k^{2}\right)^{2}+4 \delta^{2} \Delta \omega^{2} k^{2}\right]\right\}=P_{0}^{2} .
$$

Возвращаясь в пространство оригиналов, получаем разностное уравнение

$$
\tilde{a}_{k}^{2}\left[\left(p^{2}-\Delta \omega^{2} k^{2}\right)^{2}+4 \delta^{2} \Delta \omega^{2} k^{2}\right]-\tilde{a}_{k-1}^{2}\left[\left(p^{2}-\Delta \omega^{2}(k-1)^{2}\right)^{2}+4 \delta^{2} \Delta \omega^{2}(k-1)^{2}\right]=P_{0}^{2} \delta_{k},
$$

где $\delta_{k}=\left\{\begin{array}{lll}1, & \text { при } k=0, \\ 0, & \text { при } k \neq 0\end{array} \quad\right.$ символ Кронекера.

При $k=0$ получаем $\tilde{a}_{0}^{2}=\frac{P_{0}^{2}}{p^{4}}$. При $k \geqslant 1$ приходим к разностному уравнению

$$
\tilde{a}_{k}^{2}\left[\left(p^{2}-\Delta \omega^{2} k^{2}\right)^{2}+4 \delta^{2} \Delta \omega^{2} k^{2}\right]-\tilde{a}_{k-1}^{2}\left[\left(p^{2}-\Delta \omega^{2}(k-1)^{2}\right)^{2}+4 \delta^{2} \Delta \omega^{2}(k-1)^{2}\right]=0,
$$

из которого после преобразований получаем линейно параметрическую дискретную модель, связывающую два последовательных дискретных значения АЧХ:

$$
\lambda_{0}\left(k^{2} \tilde{a}_{k}^{2}-(k-1)^{2} \tilde{a}_{k-1}^{2}\right)+\lambda_{1}\left(k^{4} \tilde{a}_{k}^{2}-(k-1)^{4} a_{k-1}^{2}\right)=\tilde{a}_{k-1}^{2}-\tilde{a}_{k}^{2} .
$$


Коэфрициенты в модели (2) связаны с параметрами АЧХ следующими соотношениями:

$$
\lambda_{0}=\frac{2 \Delta \omega^{2}}{p^{4}}\left(2 \delta^{2}-p^{2}\right), \quad \lambda_{1}=\frac{\Delta \omega^{4}}{p^{4}}
$$

При обработке АЧХ формируется выборка результатов наблюдений $a_{k}(k=0,1, \ldots, N-1)$, где $N$ - объём выборки. Результаты наблюдений $a_{k}$ содержат аддитивную случайную помеху $\varepsilon_{k}$ : $a_{k}=\tilde{a}_{k}+\varepsilon_{k}$, где $\tilde{a}_{k}-$ значения теоретической АЧХ, удовлетворяющие соотношению (2). Обозначив $\lambda_{2}=\frac{P_{0}^{2}}{p^{4}}$, с учётом случайной помехи в результатах измерения АЧХ, линейно параметрическую дискретную модель можно представить в форме стохастических разностных уравнений:

$$
\left\{\begin{array}{l}
a_{0}^{2}=\lambda_{2}-\varepsilon_{0}^{2}+2 \varepsilon_{0} a_{0}, \\
a_{k-1}^{2}-a_{k}^{2}=\lambda_{0}\left(k^{2} a_{k}^{2}-(k-1)^{2} a_{k-1}^{2}\right)+\lambda_{1}\left(k^{4} a_{k}^{2}-(k-1)^{4} a_{k-1}^{2}\right)+\eta_{k}, k=1,2 \ldots, N-1 ; \\
\eta_{k}=\left[\lambda_{0} k^{2}+\lambda_{1} k^{4}+1\right]\left(\varepsilon_{k}^{2}-2 \varepsilon_{k} a_{k}\right)-\left[\lambda_{0}(k-1)^{2}+\lambda_{1}(k-1)^{4}+1\right]\left(\varepsilon_{k-1}^{2}-2 \varepsilon_{k-1} a_{k-1}\right), k=1,2 \ldots, N-1 .
\end{array}\right.
$$

Пренебрегая величинами второго порядка малости относительно $\varepsilon_{k}$, систему (3) можно представить в виде

$$
\left\{\begin{array}{l}
a_{0}^{2}=\lambda_{2}+\eta_{0}, \\
a_{k-1}^{2}-a_{k}^{2}=\lambda_{0}\left(k^{2} a_{k}^{2}-(k-1)^{2} a_{k-1}^{2}\right)+\lambda_{1}\left(k^{4} a_{k}^{2}-(k-1)^{4} a_{k-1}^{2}\right)+\eta_{k}, \quad k=1,2 \ldots, N-1 ; \\
\eta_{0}=2 a_{0} \varepsilon_{0}, \\
\eta_{k}=2 a_{k-1}\left[\lambda_{0}(k-1)^{2}+\lambda_{1}(k-1)^{4}+1\right] \varepsilon_{k-1}-2 a_{k}\left[\lambda_{0} k^{2}+\lambda_{1} k^{4}+1\right] \varepsilon_{k}, \quad k=1,2 \ldots, N-1 .
\end{array}\right.
$$

В матричной форме линейно параметрическая дискретная модель (4), описывающая последовательность значений АЧХ при случайной аддитивной помехе в результатах наблюдений имеет вид

$$
\left\{\begin{array}{l}
b=F \lambda+\eta \\
\eta=P_{a, \lambda} \varepsilon
\end{array}\right.
$$

Здесь $\lambda=\left(\lambda_{0}, \lambda_{1}, \lambda_{2}\right)^{\mathrm{T}}-$ вектор неизвестных коэфрфициентов ЛПдМ; $\varepsilon=\left(\varepsilon_{0}, \varepsilon_{1}, \ldots, \varepsilon_{N-1}\right)^{\mathrm{T}}-N$-мерный вектор случайной помехи в результатах наблюдений; $\eta=\left(\eta_{0}, \eta_{1}, \ldots, \eta_{N-1}\right)^{\mathrm{T}}-N$-мерный вектор эквивалентного возмущения в стохастическом разностном уравнении; $b=\left(a_{0}^{2}, a_{0}^{2}-a_{1}^{2}, \ldots\right.$, $\left.a_{N-2}^{2}-a_{N-1}^{2}\right)^{\mathrm{T}}-N$-мерный вектор правой части; $F$ - матрица регрессоров размера $N \times 3$, столбцы которой описываются следующими фрормулами:

$$
\begin{gathered}
f_{i 1}=\left(0, a_{1}^{2}, 4 a_{2}^{2}-a_{1}^{2}, \ldots,(N-1)^{2} a_{N-1}^{2}-(N-2)^{2} a_{N-2}^{2}\right)^{\mathrm{T}}, \\
f_{i 2}=\left(0, a_{1}^{2}, 16 a_{2}^{2}-a_{1}^{2}, \ldots,(N-1)^{4} a_{N-1}^{2}-(N-2)^{4} a_{N-2}^{2}\right)^{\mathrm{T}}, \quad f_{i 3}=(1,0, \ldots, 0)^{\mathrm{T}}, \quad i=1,2, \ldots, N .
\end{gathered}
$$

Матрица $P_{a, \lambda}$ размера $N \times N$ в стохастическом разностном уравнении эквивалентного возмущения - нижняя треугольная, ленточная, двухдиагональная. Первый столбец матрицы $P_{a, \lambda}$ имеет вид $p_{i 1}=\left(2 a_{0}, 2 a_{0}, 0, \ldots, 0\right)^{\mathrm{T}}(i=1,2, \ldots, N)$. Остальные столбцы матрицы $P_{a, \lambda}(j=2,3, \ldots, N)$ описываются так:

$$
p_{i j}=\left\{\begin{array}{cl}
0, & i<j \text { и } i>j+1 \\
-2 a_{i-1}\left[\lambda_{0}(i-1)^{2}+\lambda_{1}(i-1)^{4}+1\right], & i=j, \\
-p_{i-1, j}, & i=j+1 .
\end{array}\right.
$$

Построенная в форме стохастических разностных уравнений, линейно параметрическая дискретная модель служит основой нового, помехозащищённого метода определения динамических характеристик систем с линейно-вязким трением по их амплитудно-частотной характеристике. Этот метод включает следующие основные этапы.

1. Формирование выборки $a_{k}(k=1,2, \ldots, N-1)$ результатов измерений с шагом $\Delta \omega$ амплитудно-частотной характеристики системы, где $N$ - объем выборки.

2. Вычисление по приведённым выше формулам элементов матрицы $F$ и вектора $b$.

3. Среднеквадратичное оценивание коэффициентов $\lambda_{i}(i=0,1,2)$ из первого стохастического разностного уравнения системы (5). При этом наиболее эффективным является итерационный 
метод среднеквадратичного оценивания коэффициентов линейно параметрической дискретной модели [2]. В соответствии с этим методом вначале вычисляются МНК-оценки $\hat{\lambda}^{(0)}=\left(F^{T} F\right)^{-1} F^{T} b$, на основе которых формируется $\hat{P}_{a, \lambda}^{(1)}$ - первое приближение матрицы $P_{a, \lambda}$. Затем первое уравнение в системе (5) преобразуется к виду $\left(\hat{P}_{a, \lambda}^{(1)}\right)^{-1} b=\left(\hat{P}_{a, \lambda}^{(1)}\right)^{-1} F \lambda+\varepsilon$. Вновь вычисляются Мнкоценки, но уже для преобразованного уравнения. Процедура уточнения повторяется несколько раз.

4. Вычисление на основе среднеквадратичных оценок $\hat{\lambda}_{i}$ параметров системы с линейновязким трением по следующим формулам:

$$
p=\frac{\Delta \omega}{\sqrt[4]{\hat{\lambda}_{1}}}, \quad \delta=\frac{1}{2 \sqrt[4]{\hat{\lambda}_{1}}} \sqrt{\hat{\lambda}_{0} p^{2}+2 \Delta \omega^{2}}, \quad P_{0}=p^{2} \sqrt{\hat{\lambda}_{2}} .
$$

Проведены численно-аналитические исследования эффективности метода определения динамических характеристик на основе среднеквадратичного оценивания коэффициентов стохастического разностного уравнения по сравнению с методом кривой резонанса. Численный эксперимент был организован следующим образом. Генерировалась выборка $\tilde{a}_{k}(k=0,1, \ldots, N-1)$ дискретных значений функции, описывающей АЧХ системы с линейно-вязким трением с параметрами $a_{0}=1, \delta_{0}=0,05, p=2 \pi$. Период дискретизации $\Delta \omega$ был выбран таким образом, что при $T=\frac{2 \pi}{p}=1$ отношение $\frac{\Delta \omega}{T}=0,1$. Объём выборки составил $N=100$. В отсчёты $\tilde{a}_{k}$, соответствующие точным значениям функции, добавлялась аддитивная помеха $\varepsilon_{k}$, значения которой обеспечивали заданную величину мощности помехи от $0 \%$ до $5 \%$ в относительных единицах: $\frac{\|\varepsilon\|}{\|\tilde{a}\|} 100 \%$. Число усреднений $M=100$. При использовании метода кривой резонанса уровень измерения ширины пика выбирался равным $\alpha=\frac{\sqrt{2}}{2}$ [1].

На рис. 1 представлены зависимости относительной погрешности вычисления декремента колебаний от величины случайной помехи в результатах наблюдений. Точки 1 соответствуют применению известного метода кривой резонанса [1], а точки 2 - вычислениям на основе итерационной процедуры среднеквадратичного оценивания коэффициентов стохастического разностного уравнения. На рис. 2 представлены соответствующие зависимости дисперсий оценок декремента колебаний.

Таким образом, построена линейно параметрическая дискретная модель, описывающая в форме стохастических разностных уравнений результаты наблюдений амплитудно-частотной характеристики механической системы с линейно-вязким трением. Разработанный на основе этой модели помехозащищённый метод определения декремента колебаний системы позволяет в несколько раз повысить точность оценивания по сравнению с известным методом определения декремента колебаний по ширине пика АЧХ.

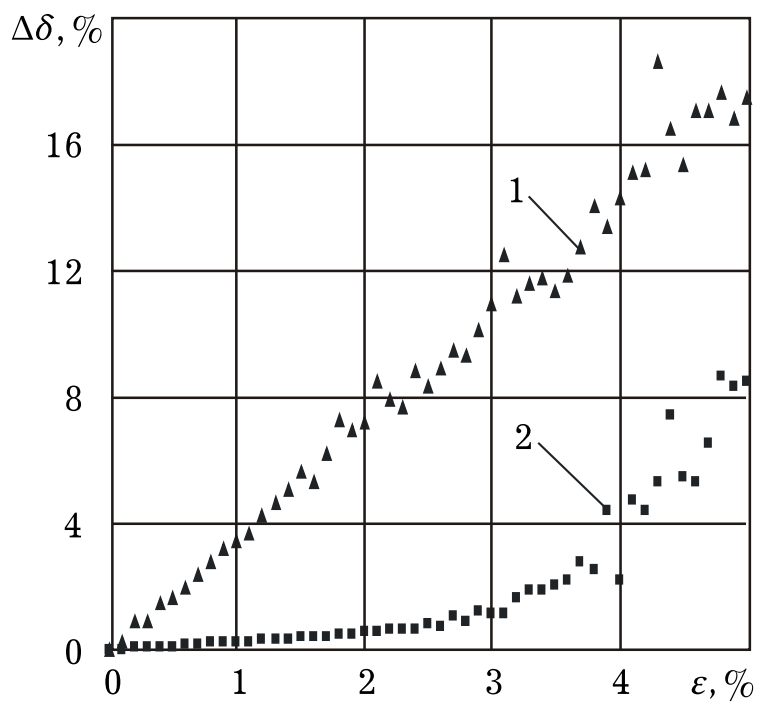

Рис. 1. Зависимости относительной погрешности вычисления декремента колебаний от величины случайной помехи в результатах наблюдений

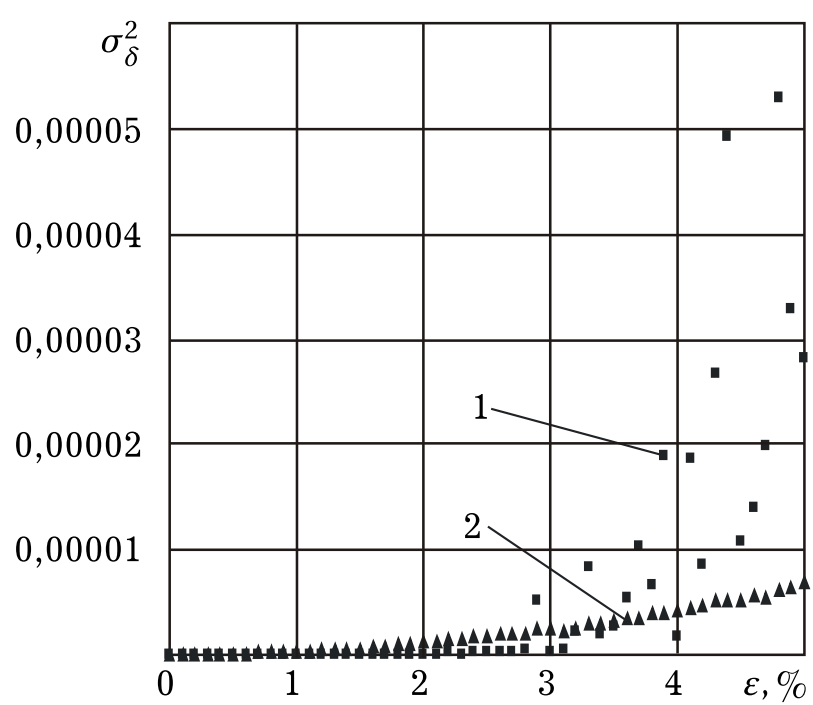

Рис. 2. Зависимости дисперсий оценок декремента колебаний от величины случайной помехи в результатах наблюдений 
Вестн. Сам. гос. техн. ун-та. Сер.: Физ.-мат. науки. - 2007. - № 2(15). - С. 182-183. - ISSN 1991-8615

\section{БИБЛИОГРАФИЧЕСКИЙ СПИСОК}

1. Писаренко, Г.С. Вибропоглощающие свойства конструкционных материалов [Текст]: Справочник / Г. С. Писаренко, А. П. Яковлев, В. В. Матвеев. - Киев: Наук. думка, 1971. - С. 36-45.

2. Зотеев, В.Е. Определение динамических характеристик нелинейных диссипативных систем на основе стохастического разностного уравнения [Текст] / В.Е. Зотеев, Д. Н. Попова // Вестн. Сам. госуд. техн. ун-та. Сер.: Физ.-мат. науки. - 2006. - № 42. - С. 162-168. - ISBN 5-7964-0815-1.

3. Пановко, Я.Г. Основы прикладной теории колебаний и удара [Текст] / Я.Г. Пановко. - Л.: Машиностроение, 1976. $-320 \mathrm{c}$.

\section{УДК 519.876.2}

\section{А. В. Докучаев, А.П. Котенко \\ РЕШЕНИЕ ЗАДАЧИ КАЛЕНДАРНОГО ПЛАНИРОВАНИЯ ПРОИЗВОДСТВА В УСЛОВИЯХ СТОХАСТИЧЕСКОЙ НЕОПРЕДЕЛЁННОСТИ ПАРАМЕТРОВ}

Приводятся новые зависимости для стохастических задач планирования производтва, полученные на основе разработанного програмлного комплекса при дискретном распределении ограниченного ресурса, и предлагаются методъ их использования.

Рассматривается задача календарного планирования производства в условиях неопределённости исходных данных при дискретном распределении ограниченного ресурса. Для решения этой задачи на основе методов динамического программирования $[1,2]$ разработан программный комплекс.

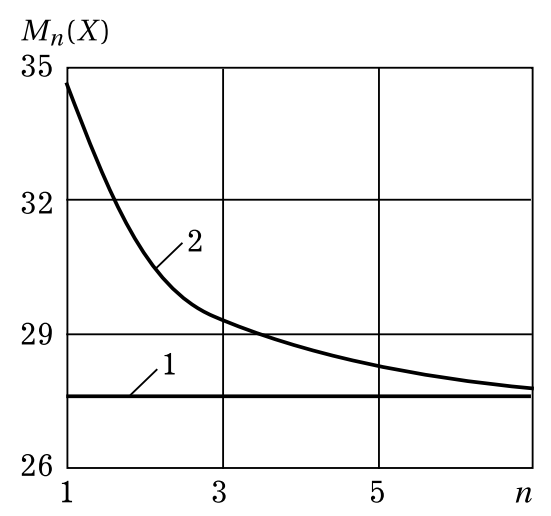

Рис. 1. Влияние числа точек спектра $n$ на математическое ожидание суммарного эфрфекта $M_{n}(X)$ : 1 - истинное значение математического ожидания, 2-математическое ожидание, получаемое без учёта стохастических особенностей задачи
Проведённые с помощью программного комплекса экспериментальные исследования позволили получить новые зависимости для стохастических задач планирования: изучено влияние шага дискретизации ресурса, числа стохастических параметров задачи, числа точек спектра и вида распределения на математическое ожидание суммарного эффекта.

Решалась задача распределения ограниченного ресурса (например, денег) по периодам освоения (например, временным промежуткам). Построены и рассмотрены две модели задачи: детерминированная модель, получаемая усреднением значений функций освоения, и стохастическая модель, учитывающая все стохастические особенности задачи. На рис. 1 представлены решения, полученные в ходе реализации предложенных алгоритмов.

Установлено, что математическое ожидание суммарного эфффекта $M_{n}(X)$ при росте числа точек спектра функций освоения $n$ стремится к истинному значению математического ожидания: $\lim _{n \rightarrow \infty} M_{n}(X) \rightarrow M(X)(n \in \mathbb{N})$.

Математическое ожидание суммарного эффекта, которое получается при увеличении числа спектральных точек, более достоверно, чем при усреднении исходных данных без учёта особенностей их стохастических распределений. То есть, эффект от распределения ресурсов без учёта неопределенности исходных данных может быть завышен, и, например, предприятие рискует не получить планируемую максимальную прибыль.

В работе предложен метод оценки риска принятия приближенного решения для случая, когда распределяемой величиной являются денежные ресурсы, а функцией освоения - временные промежутки:

$$
\text { Risk }=\frac{M(X)_{\max }-M(X)_{\min }}{M(X)_{\min }} \cdot 100 \%,
$$

где Risk - это риск принятия ошибочного решения; $M(X)_{\max }$ - математическое ожидание сум- 